\title{
Strong converse inequality for left Bernstein-Durrmeyer quasi-interpolants
}

Shunsheng GuO ${ }^{1,2}$, Guofen Liu ${ }^{1,2^{*}}$ and Xiuzhong Yang ${ }^{1,2}$

${ }^{*}$ Correspondence:

liugf2003@163.com

${ }^{1}$ College of Mathematics and

Information Science, Hebei Normal

University, Shijiazhuang, 050024,

People's Republic of China

${ }^{2}$ Hebei Key Laboratory of

Computational Mathematics and

Applications, Shijiazhuang, 050024,

People's Republic of China

\section{Abstract}

For the left Bernstein-Durrmeyer quasi-interpolants $M_{n}^{(2 r-1)} f$, we prove that, for some I,

$$
\omega_{\varphi}^{2 r}\left(f, \frac{1}{\sqrt{n}}\right)_{p} \leq C\left(\left\|M_{n}^{(2 r-1)} f-f\right\|_{p}+\left\|M_{l n}^{(2 r-1)} f-f\right\|_{p}\right) \quad(1<p \leq \infty) .
$$

This is a strong converse inequality of type B.

MSC: 41A27; 41A40

Keywords: strong converse inequality; modulus of smoothness; $K$-functional; Bernstein-Durrmeyer left quasi-interpolants

\section{Introduction}

For $f \in L_{p}[0,1]$ the Bernstein-Durrmeyer operators are given by

$$
M_{n}(f, x)=(n+1) \sum_{k=0}^{n} p_{n, k}(x) \int_{0}^{1} p_{n, k}(t) f(t) d t
$$

where $p_{n, k}(x)=\left(\begin{array}{l}n \\ k\end{array}\right) x^{k}(1-x)^{n-k}(c f .[1,2]$ and $[3,4]$ for more integral type operators). The rate of convergence and the inverse theorem for $M_{n}(f, x)$ and their combination have been investigated in [5]. Recently Sablonnière $(c f .[6,7])$ introduced a family of operators, socalled quasi-interpolants. Many quasi-interpolants of different operators were studied (e.g. [8-12]).

In the following $\Pi_{j}$ denotes the space of algebraic polynomials of degree at most $j$. Because $M_{n}$ is an automorphism of $\Pi_{n}, M_{n}$ and its inverse $M_{n}^{-1}$ can be expressed as linear differential operators with polynomial coefficients in the forms $M_{n}=\sum_{j=0}^{n} \beta_{j}^{n}(x) D^{j}$ and $M_{n}^{-1}=\sum_{j=0}^{n} \alpha_{j}^{n}(x) D^{j}$, where $D^{0}=i d, D=\frac{d}{d x}$. The polynomials $\alpha_{j}^{n}(x) \in \Pi_{j}$ are expressed explicitly in terms of shifted Jacobi polynomials $(c f .[6,9,12])$ as

$$
\alpha_{j}^{n}(x)=\sum_{s=0}^{[j / 2]}(-1)^{s} X^{s} J_{j-2 s}^{(s, s)}(x) / s !(n)_{j-s},
$$

where $X=x(1-x),(n)_{j}=n(n-1) \cdots(n+j-1)$, and

$$
J_{j-2 s}^{(s, s)}(x)=\sum_{i=0}^{j-2 s}\left(\begin{array}{c}
j-s \\
i
\end{array}\right)\left(\begin{array}{c}
j-s \\
j-2 s-i
\end{array}\right)(x-1)^{j-2 s-i} x^{i} .
$$

(c) 2015 Guo et al. This article is distributed under the terms of the Creative Commons Attribution 4.0 International License (http://creativecommons.org/licenses/by/4.0/), which permits unrestricted use, distribution, and reproduction in any medium, provided you give appropriate credit to the original author(s) and the source, provide a link to the Creative Commons license, and indicate if changes were made. 
Now we give the definition of left Bernstein-Durrmeyer quasi-interpolants $(c f .[6,9,12])$ :

$$
M_{n}^{(r)}(f, x)=\sum_{j=0}^{r} \alpha_{j}^{n}(x) D^{j} M_{n}(f, x)=: \sum_{j=0}^{r} \alpha_{j}^{n}(x) M_{n, j}(f, x),
$$

where $M_{n, j}=D^{j} M_{n}$. It is well known that $\alpha_{0}^{n}(x)=1, M_{n}^{(r)}$ is exact on $\Pi_{r}$, i.e. $M_{n}^{(r)} p=p$ for all $p \in \Pi_{r}, 0 \leq r \leq n$.

For $M_{n}^{(2 r-1)}(f, x)$ the global approximation equivalent theorem has been obtained in [9] as follows.

Theorem [9] Let $f \in L_{p}[0,1], 1<p \leq \infty, \varphi(x)=\sqrt{x(1-x)}, n \geq 4 r, r \in N, 0<\alpha<r$, then

$$
\left\|M_{n}^{(2 r-1)} f-f\right\|_{p}=O\left(n^{-\alpha}\right) \quad \Leftrightarrow \quad \omega_{\varphi}^{2 r}(f, t)_{p}=O\left(t^{2 \alpha}\right) .
$$

Here

$$
\begin{aligned}
& \omega_{\varphi}^{s}(f, t)_{p}=\sup _{0<h \leq t}\left\|\triangle_{h \varphi}^{s} f(x)\right\|_{p} \\
& \triangle_{h \varphi}^{s} f(x)=\sum_{k=0}^{s}(-1)^{k}\left(\begin{array}{l}
s \\
k
\end{array}\right) f(x+s h / 2-k h) .
\end{aligned}
$$

This is Ditzian-Totik modulus of smoothness, it is equivalent to $K$-functional

$$
K_{\varphi}^{s}\left(f, t^{s}\right)_{p}=\inf _{g \in W^{s}(\varphi)}\left\{\|f-g\|_{p}+t^{s}\left\|\varphi^{s} g^{(s)}\right\|_{p}\right\}
$$

where $W^{s}(\varphi)=:\left\{g \in L_{p}[0,1], g^{(s-1)} \in A . C \cdot[0,1],\left\|\varphi^{s} g^{(s)}\right\|_{p}<\infty\right\}$. It was proved that $\omega_{\varphi}^{s}(f, t)_{p} \sim$ $K_{\varphi}^{s}\left(f, t^{s}\right)_{p}$, i.e. there exists $A>0$ such that (cf. [13])

$$
A^{-1} \omega_{\varphi}^{s}(f, t)_{p} \leq K_{\varphi}^{s}\left(f, t^{s}\right)_{p} \leq A \omega_{\varphi}^{s}(f, t)_{p} .
$$

The strong converse inequality is an important problem of operator approximation theory. The strong converse inequalities for various operators have been investigated in subsequent papers $(e . g .[14,15])$. In most of these results the second order moduli of smoothness $\omega_{\varphi}^{2}(f, t)_{p}$ were used. The intention of this paper is to prove a strong converse inequality of type B for the quasi-interpolants $M_{n}^{(2 r-1)} f$ by using high order modulus. To this end we have to prove several key lemmas presented in Section 2. Application of these lemmas enables us to prove our main result in Section 3.

Throughout this paper $C$ denotes a positive constant independent of $n$ and $x$ not necessarily the same at each occurrence.

\section{Lemmas}

In this section we give some lemmas.

Lemma $2.1(c f .[9,10])$ For $j \geq 1, r \in N$, we have

$$
\left|\alpha_{j}^{n}(x)\right| \leq C n^{-\frac{j}{2}} \delta_{n}^{j}(x), \quad\left|D^{r} \alpha_{j}^{n}(x)\right| \leq C n^{\frac{-j+r}{2}} \delta_{n}^{j-r}(x),
$$

where $\delta_{n}(x)=\varphi(x)+\frac{1}{\sqrt{n}} \sim \max \left\{\varphi(x), \frac{1}{\sqrt{n}}\right\}$. 
Lemma 2.2 Let $E_{n}=\left[\frac{1}{n}, 1-\frac{1}{n}\right], \varphi(x)=\sqrt{x(1-x)}, f \in W^{2 r+1}(\varphi)$ and $R_{2 r+1}(f, t, x)=\frac{1}{(2 r) !} \int_{x}^{t}(t-$ $u)^{2 r} f^{(2 r+1)}(u) d u$, then we have, for $1<p \leq \infty$,

$$
\left\|M_{n}^{(2 r-1)}\left(R_{2 r+1}(f, \cdot, x), x\right)\right\|_{p}^{E_{n}} \leq C n^{-r-\frac{1}{2}}\left\|\varphi^{2 r+1} f^{(2 r+1)}\right\|_{p} .
$$

Proof Let $\psi(u)=\varphi^{2 r+1}(u) f^{(2 r+1)}(u), G(x)=M(\psi, x)=\sup _{t}\left|\frac{1}{t-x} \int_{x}^{t}\right| \psi(u)|d u|$, i.e. $G(x)$ is the maximal function of $\psi$. Noting that (cf. [7])

$$
\left|D^{j} p_{n, k}(x)\right| \leq C \sum_{i=0}^{j}\left(\frac{\sqrt{n}}{\varphi(x)}\right)^{j+i}\left|\frac{k}{n}-x\right|^{i} p_{n, k}(x), \quad x \in E_{n}
$$

we have, for $x \in E_{n}$,

$$
\left|D^{j} M_{n}(f, x)\right|=\left|M_{n, j}(f, x)\right| \leq C \sum_{i=0}^{j}\left(\frac{\sqrt{n}}{\varphi(x)}\right)^{j+i} \sum_{k=0}^{n} p_{n, k}(x)\left|\frac{k}{n}-x\right|^{i}\left|a_{k}(n)\right|,
$$

where $a_{k}(n)=(n+1) \int_{0}^{1} p_{n, k}(t) f(t) d t$. So, for $x \in E_{n}$,

$$
\begin{aligned}
& \left|M_{n}^{(2 r-1)}\left(R_{2 r+1}(f, \cdot, x), x\right)\right| \\
& \quad \leq \sum_{j=0}^{2 r-1}\left|\alpha_{j}^{n}(x)\right|\left|M_{n, j}\left(R_{2 r+1}(f, \cdot, x), x\right)\right| \\
& \quad \leq C \sum_{j=0}^{2 r-1}\left|\alpha_{j}^{n}(x)\right| \sum_{i=0}^{j}\left(\frac{\sqrt{n}}{\varphi(x)}\right)^{j+i} \sum_{k=0}^{n} p_{n, k}(x)\left|\frac{k}{n}-x\right|^{i}\left|\bar{a}_{k}(n)\right| \\
& \quad=: C \sum_{j=0}^{2 r-1} I_{j},
\end{aligned}
$$

where $\bar{a}_{k}(n)=\frac{n+1}{(2 r) !} \int_{0}^{1} p_{n, k}(t) \int_{x}^{t}(t-u)^{2 r} f^{(2 r+1)}(u) d u d t$. Using (9.6.1) in [13], we have

$$
\begin{aligned}
\left|\bar{a}_{k}(n)\right| & =\frac{n+1}{(2 r) !} \int_{0}^{1} p_{n, k}(t)\left|\int_{x}^{t} \frac{(t-u)^{2 r}}{\varphi^{2 r+1}(u)} \varphi^{2 r+1}(u) f^{(2 r+1)}(u) d u\right| d t \\
& \leq \frac{n+1}{(2 r) !} \varphi^{-(2 r+1)}(x) G(x) \int_{0}^{1} p_{n, k}(t)|t-x|^{2 r+1} d t .
\end{aligned}
$$

Hence by Hölder's inequality, we have, for $x \in E_{n}$,

$$
\begin{aligned}
\left\|I_{j}\right\|_{p}^{E_{n}} \leq & \|G(x)\|_{p} \| \alpha_{j}^{n}(x) \varphi^{-(2 r+1)}(x) \sum_{i=0}^{j}\left(\frac{\sqrt{n}}{\varphi(x)}\right)^{j+i} \sum_{k=0}^{n} p_{n, k}(x)\left|\frac{k}{n}-x\right|^{i} \\
& \times(n+1) \int_{0}^{1} p_{n, k}(t)|t-x|^{2 r+1} d t \|_{p}^{E_{n}} \\
\leq & \|G(x)\|_{p} \| \alpha_{j}^{n}(x) \varphi^{-(2 r+1)}(x) \sum_{i=0}^{j}\left(\frac{\sqrt{n}}{\varphi(x)}\right)^{j+i}\left(\sum_{k=0}^{n} p_{n, k}(x)\left(\frac{k}{n}-x\right)^{2 i}\right)^{1 / 2} \\
& \times\left(\sum_{k=0}^{n} p_{n, k}(x)(n+1) \int_{0}^{1} p_{n, k}(t)(t-x)^{4 r+2} d t\right)^{1 / 2} \|_{p}^{E_{n}} .
\end{aligned}
$$


From (9.4.14) in [13] and (6.4) in [5], we have, for $x \in E_{n}$,

$$
\begin{aligned}
& \left(\sum_{k=0}^{n} p_{n, k}(x)\left(\frac{k}{n}-x\right)^{2 i}\right)^{1 / 2} \leq C \frac{\varphi^{i}(x)}{n^{\frac{i}{2}}}, \\
& \left(\sum_{k=0}^{n} p_{n, k}(x)(n+1) \int_{0}^{1} p_{n, k}(t)(t-x)^{4 r+2} d t\right)^{1 / 2} \leq C \frac{\varphi^{2 r+1}(x)}{n^{r+\frac{1}{2}}} .
\end{aligned}
$$

Together with (2.1) and the fact that

$$
\|G(x)\|_{p} \leq C_{p}\left\|\varphi^{2 r+1} f^{(2 r+1)}\right\|_{p}
$$

we obtain (2.2).

Lemma 2.3 For $n \geq 2 r$, we have

$$
\begin{aligned}
& M_{n}^{(2 r-1)}\left((t-x)^{2 r}, x\right) \\
& =(-1)^{r+1} n^{-r} \varphi^{2 r}(x) \frac{(2 r) !}{2^{r}(r !)}+\varphi^{2 r}(x) o\left(\frac{1}{n^{r}}\right) \\
& \quad+(2 r) !\left(b_{2 r}^{n} \frac{1}{n^{2 r}}+b_{2 r-1}^{n} \frac{\varphi^{2}(x)}{n^{2 r-1}}+\cdots+b_{r+1}^{n} \frac{\varphi^{2 r-2}(x)}{n^{r+1}}\right)\left(1+O\left(\frac{1}{n}\right)\right),
\end{aligned}
$$

where $b_{j}^{n}$ are uniformly bounded in $n$ and independent of $x$.

Proof First we note $M_{n}^{(2 r)} p=p$ for all $p \in \Pi_{2 r}$, so we have

$$
M_{n}^{(2 r)}\left((t-x)^{2 r}, x\right)=0
$$

then

$$
M_{n}^{(2 r)}\left((t-x)^{2 r}, x\right)-M_{n}^{(2 r-1)}\left((t-x)^{2 r}, x\right)=\alpha_{2 r}^{n}(x) M_{n, 2 r}\left((t-x)^{2 r}, x\right) .
$$

Therefore we have

$$
M_{n}^{(2 r-1)}\left((t-x)^{2 r}, x\right)=-\alpha_{2 r}^{n}(x) M_{n, 2 r}\left((t-x)^{2 r}, x\right) .
$$

Using (cf. [5])

$$
M_{n, 2 r}(f, x)=\frac{(n+1) ! n !}{(n-2 r) !(n+2 r) !} \sum_{k=0}^{n-2 r} p_{n-2 r, k}(x) \int_{0}^{1} p_{n+2 r, k+2 r}(t) f^{(2 r)}(t) d t
$$

we have

$$
M_{n, 2 r}\left((t-x)^{2 r}, x\right)=\frac{(n+1) ! n !(2 r) !}{(n-2 r) !(n+2 r) !}=(2 r) !\left(1+O\left(\frac{1}{n}\right)\right) .
$$

Therefore

$$
M_{n}^{(2 r-1)}\left((t-x)^{2 r}, x\right)=-(2 r) ! \alpha_{2 r}^{n}(x)\left(1+O\left(\frac{1}{n}\right)\right) .
$$


Also we have $(c f .(3.11)$ in $[10])$

$$
\alpha_{2 r}^{n}(x)=b_{2 r}^{n} \frac{1}{n^{2 r}}+b_{2 r-1}^{n} \frac{\varphi^{2}(x)}{n^{2 r-1}}+\cdots+b_{r}^{n} \frac{\varphi^{2 r}(x)}{n^{r}},
$$

where $b_{j}^{n}$ are uniformly bounded in $n$ and independent of $x$.

By Theorem 4.2 and Table 2 in [7] we know that $\lim _{n} n^{r} \alpha_{2 r}^{n}(x)$ exists and

$$
\lim _{n} n^{r} \alpha_{2 r}^{n}(x)=\frac{(-1)^{r} \varphi^{2 r}(x)}{2^{r}(r !)}
$$

With this relation and (2.6), we get the representation of the coefficient $b_{r}^{n}$ in (2.6), i.e.

$$
\lim _{n} b_{r}^{n}=\frac{(-1)^{r}}{2^{r}(r !)}
$$

From (2.5)-(2.8) we get (2.3).

Lemma 2.4 For $f \in W^{2 r}(\varphi), 1<p \leq \infty$, we have

$$
\left\|\varphi^{2 r+1} D^{2 r+1}\left(M_{n}^{(2 r-1)} f\right)\right\|_{p} \leq C \sqrt{n}\left\|\varphi^{2 r} f^{(2 r)}\right\|_{p} .
$$

Proof By (2.6) in [5] one has, for $x \in[0,1], 1 \leq p \leq \infty, r, s \in N_{0}=N \cup\{0\}$,

$$
\left\|\delta_{n}^{s}(x) \varphi^{2 r}(x) D^{2 r+s} M_{n}(f, x)\right\|_{p} \leq C n^{\frac{s}{2}}\left\|\varphi^{2 r} f^{(2 r)}\right\|_{p}
$$

So we have

$$
\left\|\varphi^{2 r+m}(x) D^{2 r+m} M_{n}(f, x)\right\|_{p}^{E_{n}} \leq C n^{\frac{m}{2}}\left\|\varphi^{2 r} f^{(2 r)}\right\|_{p}, \quad m \geq 0 .
$$

Using (2.1) and (2.10), we have

$$
\begin{aligned}
& \left\|\varphi^{2 r+1}(x) D^{2 r+1} \sum_{j=0}^{2 r-1} \alpha_{j}^{n}(x) M_{n, j}(f, x)\right\|_{p}^{E_{n}} \\
& \leq \sum_{j=0}^{2 r-1} \sum_{i=0}^{j}\left\|\varphi^{2 r+1}(x)\left(\begin{array}{c}
2 r+1 \\
i
\end{array}\right)\left(D^{i} \alpha_{j}^{n}(x)\right) M_{n, 2 r+1+j-i}(f, x)\right\|_{p}^{E_{n}} \\
& \leq C \sum_{j=0}^{2 r-1} \sum_{i=0}^{j}\left\|\varphi^{2 r+1}(x) n^{\frac{-j+i}{2}} \varphi^{j-i}(x) M_{n, 2 r+1+j-i}(f, x)\right\|_{p}^{E_{n}} \\
& \leq C \sqrt{n}\left\|\varphi^{2 r} f^{(2 r)}\right\|_{p} .
\end{aligned}
$$

Since $\left(\varphi^{2 r+1}(x) D^{2 r+1} M_{n}^{(2 r-1)}(f, x)\right)^{2}$ are polynomials, we can use a result of the weight polynomial approximation [13], Theorem 8.4 .8 , translating the interval $[-1,1]$ to $[0,1]$ to obtain the estimate

$$
\left\|\left(\varphi^{2 r+1} D^{2 r+1}\left(M_{n}^{(2 r-1)} f\right)\right)^{2}\right\|_{p}^{[0,1]} \leq M\left\|\left(\varphi^{2 r+1} D^{2 r+1}\left(M_{n}^{(2 r-1)} f\right)\right)^{2}\right\|_{p}^{E_{n}},
$$

where $M$ does not depend on $n$. From (2.11) and (2.12) we obtain (2.9). 
Lemma 2.5 ((4.2) in [9]) For $f \in L_{p}[0,1], 1 \leq p \leq \infty$, we have

$$
\left\|\varphi^{2 r} D^{2 r}\left(M_{n}^{(2 r-1)} f\right)\right\|_{p} \leq C n^{r}\|f\|_{p} .
$$

Lemma 2.6 For $f \in W^{2 r+1}(\varphi)$, we have

$$
\begin{aligned}
& M_{n}^{(2 r-1)}(f, x)-f(x)-\frac{(-1)^{r+1} \varphi^{2 r}(x)}{2^{r} n^{r}(r !)} f^{(2 r)}(x) \\
& =o\left(\frac{1}{n^{r}}\right) \varphi^{2 r}(x) f^{(2 r)}(x)+\left(b_{2 r}^{n} \frac{1}{n^{2 r}}+b_{2 r-1}^{n} \frac{\varphi^{2}(x)}{n^{2 r-1}}+\cdots+b_{r+1}^{n} \frac{\varphi^{2 r-2}(x)}{n^{r+1}}\right) \\
& \quad \times f^{(2 r)}(x)\left(1+O\left(\frac{1}{n}\right)\right)+M_{n}^{(2 r-1)}\left(R_{2 r+1}(f, \cdot, x), x\right),
\end{aligned}
$$

where $\left\{b_{2 r-1}^{n}, \ldots, b_{r+1}^{n}\right\}$ are uniformly bounded in $n$ and independent of $x$.

Proof By Taylor's formula we expand $f$ as follows:

$$
f(t)=f(x)+(t-x) f^{\prime}(x)+\cdots+\frac{(t-x)^{2 r}}{(2 r) !} f^{(2 r)}(x)+R_{2 r+1}(f, t, x),
$$

where $R_{2 r+1}(f, t, x)=\frac{1}{(2 r) !} \int_{x}^{t}(t-u)^{2 r} f^{(2 r+1)}(u) d u$.

Noting $M_{n}^{(2 r-1)} p=p$ for all $p \in \Pi_{2 r-1}(c f$. [7]), we obtain

$$
M_{n}^{(2 r-1)}(f, x)-f(x)=M_{n}^{(2 r-1)}\left(\frac{(t-x)^{2 r}}{(2 r) !}, x\right) f^{(2 r)}(x)+M_{n}^{(2 r-1)}\left(R_{2 r+1}(f, \cdot, x), x\right) .
$$

Using Lemma 2.3 we obtain (2.14).

\section{Main result}

Using the lemmas in Section 2 we are able to prove the following main result, which is the strong converse inequality for left Bernstein-Durrmeyer quasi-interpolants of type B.

Theorem 3.1 Let $f \in L_{p}[0,1], 1<p \leq \infty, \varphi(x)=\sqrt{x(1-x)}, n \geq 4 r, r \in N$, then there exists a constant $k$ such that, for $l \geq k n$,

$$
\omega_{\varphi}^{2 r}\left(f, \frac{1}{\sqrt{n}}\right)_{p} \leq C\left(\frac{l}{n}\right)^{r}\left(\left\|M_{n}^{(2 r-1)} f-f\right\|_{p}+\left\|M_{l}^{(2 r-1)} f-f\right\|_{p}\right) .
$$

Proof To prove our result at first we estimate $K$-functional $K_{\varphi}^{2 r}\left(f, n^{-r}\right)_{p}$. We choose the function

$$
g=K_{n}^{(2 r-1)}\left(K_{n}^{(2 r-1)} f\right)=: K_{n}^{2(2 r-1)} f
$$

By the definition of the $K$-functional and the boundedness of $K_{n}^{(2 r-1)}(c f$. [7], p.243, (3.2) in [9]), we have

$$
\begin{aligned}
K_{\varphi}^{2 r}\left(f, n^{-r}\right)_{p} & \leq\|f-g\|_{p}+n^{-r}\left\|\varphi^{2 r} g^{(2 r)}\right\|_{p} \\
& =\left\|f-M_{n}^{2(2 r-1)} f\right\|_{p}+n^{-r}\left\|\varphi^{2 r} D^{2 r}\left(M_{n}^{2(2 r-1)} f\right)\right\|_{p}
\end{aligned}
$$


Gro et al. Journal of Inequalities and Applications (2015) 2015:367

Page 7 of 9

$$
\begin{aligned}
\leq & \left\|f-M_{n}^{(2 r-1)} f\right\|_{p}+\left\|M_{n}^{(2 r-1)} f-M_{n}^{2(2 r-1)} f\right\|_{p} \\
& +n^{-r}\left\|\varphi^{2 r} D^{2 r}\left(M_{n}^{2(2 r-1)} f\right)\right\|_{p} \\
\leq & C\left\|f-M_{n}^{(2 r-1)} f\right\|_{p}+n^{-r}\left\|\varphi^{2 r} D^{2 r}\left(M_{n}^{2(2 r-1)} f\right)\right\|_{p} .
\end{aligned}
$$

Therefore we only need to estimate $\varphi^{2 r} g^{(2 r)}=\varphi^{2 r} D^{2 r}\left(M_{n}^{2(2 r-1)} f\right)$. We recall Lemma 2.6 with $g=M_{n}^{2(2 r-1)} f$ in place of $f$ and $l$ in place of $n$ to obtain

$$
\begin{aligned}
& M_{l}^{(2 r-1)}(g, x)-g(x)-\frac{(-1)^{r+1} \varphi^{2 r}(x)}{2^{r} l^{r}(r !)} g^{(2 r)}(x) \\
& =o\left(\frac{1}{l^{r}}\right) \varphi^{2 r}(x) g^{(2 r)}(x)+\left(b_{2 r}^{l} \frac{1}{l^{2 r}}+b_{2 r-1}^{l} \frac{\varphi^{2}(x)}{l^{2 r-1}}+\cdots+b_{r+1}^{l} \frac{\varphi^{2 r-2}(x)}{l^{r+1}}\right) \\
& \quad \times g^{(2 r)}(x)\left(1+O\left(\frac{1}{l}\right)\right)+M_{l}^{(2 r-1)}\left(R_{2 r+1}(g, \cdot, x), x\right) .
\end{aligned}
$$

For $x \in E_{n}, n \varphi^{2}(x) \geq 1$. So we have

$$
\begin{aligned}
& \left\|\frac{1}{l^{2 r}} g^{(2 r)}(x)\right\|_{p}^{E^{n}}=\left\|\frac{n^{r} \varphi^{2 r}(x)}{l^{2 r} n^{r} \varphi^{2 r}(x)} g^{(2 r)}(x)\right\|_{p}^{E^{n}} \leq \frac{1}{l^{r}}\left(\frac{n}{l}\right)^{r}\left\|\varphi^{2 r} g^{(2 r)}\right\|_{p^{\prime}} \\
& \left\|\frac{\varphi^{2}(x)}{l^{2 r-1}} g^{(2 r)}(x)\right\|_{p}^{E^{n}}=\left\|\frac{n^{r-1} \varphi^{2 r}(x)}{l^{2 r-1} n^{r-1} \varphi^{2 r-2}(x)} g^{(2 r)}(x)\right\|_{p}^{E^{n}} \leq \frac{1}{l^{r}}\left(\frac{n}{l}\right)^{r-1}\left\|\varphi^{2 r} g^{(2 r)}\right\|_{p}, \\
& \ldots, \\
& \left\|\frac{\varphi^{2 r-2}(x)}{l^{r+1}} g^{(2 r)}(x)\right\|_{p}^{E^{n}}=\left\|\frac{n \varphi^{2 r}(x)}{l^{r+1} n \varphi^{2}(x)} g^{(2 r)}(x)\right\|_{p}^{E^{n}} \leq \frac{1}{l^{r}}\left(\frac{n}{l}\right)\left\|\varphi^{2 r} g^{(2 r)}\right\|_{p} .
\end{aligned}
$$

By Lemma 2.2 we have

$$
\left\|M_{l}^{(2 r-1)}\left(R_{2 r+1}(g, \cdot, x), x\right)\right\|_{p}^{E_{n}} \leq C l^{-r-\frac{1}{2}}\left\|\varphi^{2 r+1} g^{(2 r+1)}\right\|_{p} .
$$

Combining (3.1)-(3.3), we obtain

$$
\begin{aligned}
& \frac{1}{2^{r} l r(r !)}\left\|\varphi^{2 r} g^{(2 r)}\right\|_{p}^{E_{n}} \\
& \leq\left\|M_{l}^{(2 r-1)} g-g\right\|_{p}+o\left(\frac{1}{l^{r}}\right)\left\|\varphi^{2 r} g^{(2 r)}\right\|_{p} \\
& \quad+C \frac{1}{l^{r}}\left[\left(\frac{n}{l}\right)^{r}+\left(\frac{n}{l}\right)^{r-1}+\cdots+\frac{n}{l}\right]\left(1+O\left(\frac{1}{l}\right)\right)\left\|\varphi^{2 r} g^{(2 r)}\right\|_{p} \\
& \quad+C l^{-r-\frac{1}{2}}\left\|\varphi^{2 r+1} g^{(2 r+1)}\right\|_{p} .
\end{aligned}
$$

Next we estimate the first term and the last term of the right side in (3.4). By the boundedness of $M_{n}^{(2 r-1)} f$ we have

$$
\begin{aligned}
\left\|M_{l}^{(2 r-1)} g-g\right\|_{p} & =\left\|M_{l}^{(2 r-1)}\left(M_{n}^{2(2 r-1)} f\right)-M_{n}^{2(2 r-1)} f\right\|_{p} \\
& \leq\left\|M_{l}^{(2 r-1)}\left(M_{n}^{2(2 r-1)} f-M_{n}^{(2 r-1)} f\right)\right\|_{p}+\left\|M_{l}^{(2 r-1)}\left(M_{n}^{(2 r-1)} f-f\right)\right\|_{p}
\end{aligned}
$$


Gro et al. Journal of Inequalities and Applications (2015) 2015:367

Page 8 of 9

$$
\begin{aligned}
& +\left\|M_{l}^{(2 r-1)} f-f\right\|_{p}+\left\|f-M_{n}^{(2 r-1)} f\right\|_{p}+\left\|M_{n}^{(2 r-1)}\left(f-M_{n}^{(2 r-1)} f\right)\right\|_{p} \\
\leq & C\left(\left\|M_{n}^{(2 r-1)} f-f\right\|_{p}+\left\|M_{l}^{(2 r-1)} f-f\right\|_{p}\right) .
\end{aligned}
$$

Using (2.9) and (2.13), we obtain

$$
\begin{aligned}
\left\|\varphi^{2 r+1} g^{(2 r+1)}\right\|_{p} & =\left\|\varphi^{2 r+1} D^{2 r+1}\left(M_{n}^{2(2 r-1)} f\right)\right\|_{p} \\
& \leq C \sqrt{n}\left\|\varphi^{2 r} D^{2 r}\left(M_{n}^{(2 r-1)} f\right)\right\|_{p} \\
& \leq C \sqrt{n}\left(\left\|\varphi^{2 r} D^{2 r}\left(M_{n}^{2(2 r-1)} f\right)\right\|_{p}+\left\|\varphi^{2 r} D^{2 r}\left(M_{n}^{(2 r-1)}\left(M_{n}^{(2 r-1)} f-f\right)\right)\right\|_{p}\right) \\
& \leq C \sqrt{n}\left(\left\|\varphi^{2 r} g^{(2 r)}\right\|_{p}+n^{r}\left\|M_{n}^{(2 r-1)} f-f\right\|_{p}\right) .
\end{aligned}
$$

Therefore with (3.4)-(3.6) we get

$$
\begin{aligned}
& \frac{1}{2^{r} l^{r}(r !)}\left\|\varphi^{2 r} g^{(2 r)}\right\|_{p}^{E_{n}} \\
& \leq C\left(\left\|M_{n}^{(2 r-1)} f-f\right\|_{p}+\left\|M_{l}^{(2 r-1)} f-f\right\|_{p}\right) \\
& \quad+C\left(\frac{n}{l}\right)^{r+\frac{1}{2}}\left\|M_{n}^{(2 r-1)} f-f\right\|_{p}+C l^{-r}\left(\frac{n}{l}\right)^{\frac{1}{2}}\left\|\varphi^{2 r} g^{(2 r)}\right\|_{p} \\
& \quad+C \frac{1}{l^{r}}\left[\left(\frac{n}{l}\right)^{r}+\left(\frac{n}{l}\right)^{r-1}+\cdots+\frac{n}{l}+o(1)\right]\left\|\varphi^{2 r} g^{(2 r)}\right\|_{p} .
\end{aligned}
$$

Since $\varphi^{2 r} g^{(2 r)}=\varphi^{2 r} D^{2 r}\left(M_{n}^{2(2 r-1)} f\right)$ are polynomials, for the same reason as (2.12) we have

$$
\left\|\varphi^{2 r} g^{(2 r)}\right\|_{p}^{[0,1]} \leq M\left\|\varphi^{2 r} g^{(2 r)}\right\|_{p}^{E_{n}}
$$

where $M$ does not depend on $n$. Hence by (3.7) and (3.8) we obtain

$$
\begin{aligned}
& \frac{1}{2^{r} l^{r}(r !)}\left\|\varphi^{2 r} g^{(2 r)}\right\|_{p} \\
& \leq \frac{M}{2^{r} l^{r}(r !)}\left\|\varphi^{2 r} g^{(2 r)}\right\|_{p}^{E_{n}} \\
& \leq C M\left(\left\|M_{n}^{(2 r-1)} f-f\right\|_{p}+\left\|M_{l}^{(2 r-1)} f-f\right\|_{p}\right)+C M\left(\frac{n}{l}\right)^{r+\frac{1}{2}}\left\|M_{n}^{(2 r-1)} f-f\right\|_{p} \\
& \quad+C M \frac{1}{l^{r}}\left[\left(\frac{n}{l}\right)^{r}+\left(\frac{n}{l}\right)^{r-1}+\cdots+\frac{n}{l}+\left(\frac{n}{l}\right)^{\frac{1}{2}}+o(1)\right]\left\|\varphi^{2 r} g^{(2 r)}\right\|_{p} .
\end{aligned}
$$

We now choose $l \geq k n$ with $k$ large enough such that

$$
C M \frac{1}{l^{r}}\left[\left(\frac{n}{l}\right)^{r}+\left(\frac{n}{l}\right)^{r-1}+\cdots+\frac{n}{l}+\left(\frac{n}{l}\right)^{\frac{1}{2}}+o(1)\right] \leq \frac{1}{2 \cdot 2^{r} l^{r}(r !)} .
$$

By (3.9) and (3.10) we get

$$
\frac{1}{2 \cdot 2^{r} l^{r}(r !)}\left\|\varphi^{2 r} g^{(2 r)}\right\|_{p} \leq C\left\{\left\|M_{n}^{(2 r-1)} f-f\right\|_{p}+\left\|M_{l}^{(2 r-1)} f-f\right\|_{p}\right\} .
$$


Therefore

$$
\begin{aligned}
K_{\varphi}^{2 r}\left(f, n^{-r}\right)_{p} & \leq C\left\|f-M_{n}^{(2 r-1)} f\right\|_{p}+n^{-r}\left\|\varphi^{2 r} g^{(2 r)}\right\|_{p} \\
& \leq C\left\|f-M_{n}^{(2 r-1)} f\right\|_{p}+C\left(\frac{l}{n}\right)^{r}\left(\left\|M_{n}^{(2 r-1)} f-f\right\|_{p}+\left\|M_{l}^{(2 r-1)} f-f\right\|_{p}\right) \\
& \leq C\left(\frac{l}{n}\right)^{r}\left(\left\|M_{n}^{(2 r-1)} f-f\right\|_{p}+\left\|M_{l}^{(2 r-1)} f-f\right\|_{p}\right) .
\end{aligned}
$$

\section{With (1.3) we obtain}

$$
\omega_{\varphi}^{2 r}\left(f, \frac{1}{\sqrt{n}}\right)_{p} \leq C\left(\frac{l}{n}\right)^{r}\left(\left\|M_{n}^{(2 r-1)} f-f\right\|_{p}+\left\|M_{l}^{(2 r-1)} f-f\right\|_{p}\right)
$$

\section{The proof is complete.}

\section{Competing interests}

The authors declare that they have no competing interests.

\section{Authors' contributions}

All authors conceived of the study, participated its design and coordination, drafted the manuscript, participated in the sequence alignment, and read and approved the final manuscript.

\section{Acknowledgements}

This work was supported by the National Natural Science Foundation of China (Grant Nos. 11371119 and 11401162) and by the Natural Science Foundation of Education Department of Hebei Province (Grant No. Z2014031).

\section{Received: 13 August 2015 Accepted: 9 November 2015 Published online: 21 November 2015}

\section{References}

1. Gupta, V: Approximation properties by Bernstein-Durrmeyer type operators. Complex Anal. Oper. Theory 7, 363-374 (2013)

2. Gupta, V, López-Moreno, AJ, Latorre-Palacios, JM: On simultaneous approximation of the Bernstein Durrmeyer operators. Appl. Math. Comput. 213, 112-120 (2009)

3. Srivastava, HM, Gupta, V: A certain family of summation integral type operators. Math. Comput. Model. 37, 1307-1315 (2003)

4. Gupta, V, Yadav, R: Approximation by complex summation-integral type operator in compact disks. Math. Slovaca 63(5), 1025-1036 (2013)

5. Ditzian, Z, Ivanov, KG: Bernstein-type operators and their derivatives. J. Approx. Theory 56(1), $72-90$ (1989)

6. Sablonnière, P: A family of Bernstein quasi-interpolants on [0, 1]. Approx. Theory Appl. 8(3), $62-76$ (1992)

7. Sablonnière, P: Representation of quasi-interpolants as differential operators and applications. In: Müller, MW, Buhmann, M, Mache, DH, Felten, M (eds.) New Developments in Approximation Theory. International Series of Numerical Mathematics, vol. 132, pp. 233-253. Birkhäuser, Basel (1998)

8. Diallo, AT: Rate of convergence of Bernstein quasi-interpolants. ICTP preprint IC/95/295, Miramare-Trieste, Italy (1995)

9. Duan, $L, L i, C:$ The global approximation by left Bernstein-Durrmeyer quasi-interpolants in $L_{p}[0,1]$. Anal. Theory Appl. 20(3), 242-251 (2004)

10. Guo, S, Zhang, G, Qi, Q, Liu, L: Approximation by Bernstein-Durrmeyer quasi-interpolants. Acta Math. Sin. 48(4), 681-692 (2005)

11. Guo, S, Zhang, G, Qi, Q, Liu, L: Pointwise approximation by Bernstein quasi-interpolants. Numer. Funct. Anal. Optim. 24, 339-350 (2003)

12. Mache, P, Mache, DH: Approximation by Bernstein quasi-interpolants. Numer. Funct. Anal. Optim. 22(1-2), 159-175 (2001)

13. Ditzian, Z, Totik, V: Moduli of Smoothness. Springer, New York (1987)

14. Chen, W, Ditzian, Z: Strong converse inequality for Kantorovich polynomials. Constr. Approx. 10, 95-106 (1994)

15. Ditzian, Z, Ivanov, KG: Strong converse inequalities. J. Anal. Math. 61, 61-111 (1993) 